\title{
Existence results for impulsive fractional $q$-difference equations with anti-periodic boundary conditions
}

\author{
Bashir Ahmad ${ }^{1 *}$, Jessada Tariboon², Sotiris K Ntouyas ${ }^{1,3}$, Hamed H Alsulami ${ }^{1}$ and Shatha Monaquel ${ }^{1}$
}

\author{
"Correspondence: \\ bashirahmad_qau@yahoo.com \\ ${ }^{1}$ Nonlinear Analysis and Applied \\ Mathematics (NAAM) Research \\ Group, Department of Mathematics, \\ Faculty of Science, King Abdulaziz \\ University, P.O. Box 80203, Jeddah, \\ 21589, Saudi Arabia \\ Full list of author information is \\ available at the end of the article
}

\begin{abstract}
This paper studies a Caputo type anti-periodic boundary value problem of impulsive fractional $q$-difference equations involving a $q$-shifting operator of the form ${ }_{a} \Phi_{q}(m)=q m+(1-q) a$. Concerning the existence of solutions for the given problem, two theorems are proved via Schauder's fixed point theorem and the Leray-Schauder nonlinear alternative, while the uniqueness of solutions is established by means of Banach's contraction mapping principle. Finally, we discuss some examples illustrating the main results.
\end{abstract}

MSC: 26A33; 39A13; 34A37

Keywords: quantum calculus; impulsive fractional q-difference equations; existence; uniqueness; fixed point theorem

\section{Introduction}

The quantum calculus (calculus without limits or $q$-calculus) is concerned with difference operators and allows one to deal with sets of nondifferentiable functions. Quantum difference operators appear in several branches of mathematics, such as orthogonal polynomials, basic hyper-geometric functions, combinatorics, the calculus of variations, mechanics, and the theory of relativity. For the fundamental concepts of quantum calculus, we refer the reader to the text by Kac and Cheung [1].

In recent years, the topic of $q$-calculus has attracted the attention of several researchers and a variety of new results can be found in [2-15] and the references cited therein.

In [16] and [17], the authors studied the problems involving nonlinear impulsive $q_{k}$-difference equations. In [18], some new concepts of fractional quantum calculus were introduced in terms of a $q$-shifting operator ${ }_{a} \Phi_{q}(m)=q m+(1-q) a$, and existence results for initial value problems of impulsive fractional $q$-difference equations were obtained.

In this paper we study the following anti-periodic boundary value problem of an impulsive fractional $q$-difference equation:

$$
\left\{\begin{array}{l}
{ }_{t_{k}}^{c} D_{q_{k}}^{\alpha_{k}} x(t)=f(t, x(t)), \quad t \in J_{k} \subseteq[0, T], t \neq t_{k}, \\
\Delta x\left(t_{k}\right):=x\left(t_{k}^{+}\right)-x\left(t_{k}\right)=\varphi_{k}\left(t_{k-1} I_{q_{k-1}}^{\beta_{k-1}} x\left(t_{k}\right)\right), \quad k=1,2, \ldots, m, \\
{ }_{t_{k}} D_{q_{k}} x\left(t_{k}^{+}\right)-t_{k-1} D_{q_{k-1}} x\left(t_{k}\right)=\varphi_{k}^{*}\left(t_{k-1} I_{q_{k-1}}^{\gamma_{k-1}} x\left(t_{k}\right)\right), \quad k=1,2, \ldots, m, \\
x(0)=-x(T), \quad{ }_{0} D_{q_{0}} x(0)=-{ }_{t_{m}} D_{q_{m}} x(T),
\end{array}\right.
$$

( 2016 Ahmad et al. This article is distributed under the terms of the Creative Commons Attribution 4.0 International License (http://creativecommons.org/licenses/by/4.0/, which permits unrestricted use, distribution, and reproduction in any medium, provided you give appropriate credit to the original author(s) and the source, provide a link to the Creative Commons license, and indicate if changes were made. 
where $0=t_{0}<t_{1}<\cdots<t_{m}<t_{m+1}=T,{ }_{t_{k}}^{c} D_{q_{k}}^{\alpha_{k}}$ denotes the Caputo $q_{k}$-fractional derivative of order $\alpha_{k}$ on $J_{k}, 1<\alpha_{k} \leq 2,0<q_{k}<1, J_{k}=\left(t_{k}, t_{k+1}\right], J_{0}=\left[0, t_{1}\right], k=0,1, \ldots, m, J=[0, T]$, $f \in C(J \times \mathbb{R}, \mathbb{R}), \varphi_{k}, \varphi_{k}^{*} \in C(\mathbb{R}, \mathbb{R}), k=1,2, \ldots, m, t_{k} I_{q_{k}}^{\beta_{k}},{ }_{t_{k}} I_{q_{k}}^{\gamma_{k}}$ denotes the Riemann-Liouville $q_{k}$-fractional integral of orders $\beta_{k}, \gamma_{k}>0$ on $J_{k}, k=0,1,2, \ldots, m-1$.

The rest of the paper is organized as follows. In Section 2, we present some background material for the problem at hand and prove an auxiliary lemma for the linear variant of problem (1.1). Section 3 contains the main results which are established by means of Schauder's fixed point theorem, the Leray-Schauder nonlinear alternative, and Banach's fixed point theorem. Section 4 deals with some illustrative examples for the existence results obtained in Section 3.

\section{Preliminaries}

First of all, we recall some basic concepts of $q$-calculus [18].

The $q$-derivative of a function $f$ on the interval $[a, b]$ is defined by

$$
\left({ }_{a} D_{q} f\right)(t)=\frac{f(t)-f\left({ }_{a} \Phi_{q}(t)\right)}{(1-q)(t-a)}, \quad t \neq a \quad \text { and } \quad\left({ }_{a} D_{q} f\right)(a)=\lim _{t \rightarrow a}\left({ }_{a} D_{q} f\right)(t)
$$

and $q$-derivative of higher order is given by

$$
\left({ }_{a} D_{q}^{k} f\right)(t)={ }_{a} D_{q}^{k-1}\left({ }_{a} D_{q} f\right)(t), \quad\left({ }_{a} D_{q}^{0} f\right)(t)=f(t), \quad k \in \mathbb{N} .
$$

The $q$-derivatives of a product and ratio of functions $f$ and $g$ on $[a, b]$ are

$$
\begin{aligned}
{ }_{a} D_{q}(f g)(t) & =f(t)_{a} D_{q} g(t)+g\left({ }_{a} \Phi_{q}(t)\right)_{a} D_{q} f(t) \\
& =g(t)_{a} D_{q} f(t)+f\left({ }_{a} \Phi_{q}(t)\right)_{a} D_{q} g(t)
\end{aligned}
$$

and

$$
{ }_{a} D_{q}\left(\frac{f}{g}\right)(t)=\frac{g(t)_{a} D_{q} f(t)-f(t)_{a} D_{q} g(t)}{g(t) g\left({ }_{a} \Phi_{q}(t)\right)},
$$

where $g(t) g\left({ }_{a} \Phi_{q}(t)\right) \neq 0$.

The $q$-integral of a function $f$ defined on the interval $[a, b]$ is given by

$$
\left({ }_{a} I_{q} f\right)(t)=\int_{a}^{t} f(s){ }_{a} d s=(1-q)(t-a) \sum_{i=0}^{\infty} q^{i} f\left({ }_{a} \Phi_{q^{i}}(t)\right), \quad t \in[a, b],
$$

with

$$
\left({ }_{a} I_{q}^{k} f\right)(t)={ }_{a} I_{q}^{k-1}\left({ }_{a} I_{q} f\right)(t), \quad\left({ }_{a} I_{q}^{0} f\right)(t)=f(t), \quad k \in \mathbb{N} .
$$

The fundamental theorem of calculus applies to the operators ${ }_{a} D_{q}$ and $I_{q}$ as follows:

$$
\left({ }_{a} D_{q a} I_{q} f\right)(t)=f(t) .
$$

If $f$ is continuous at $t=a$, then

$$
\left({ }_{a} I_{q a} D_{q} f\right)(t)=f(t)-f(a) .
$$


The formula for $q$-integration by parts on the interval $[a, b]$ is

$$
\int_{a}^{b} f(s)_{a} D_{q} g(s)_{a} d_{q} s=\left.(f g)(t)\right|_{a} ^{b}-\int_{a}^{b} g\left({ }_{a} \Phi_{q}(s)\right)_{a} D_{q} f(s)_{a} d_{q} s .
$$

Now we enlist some useful properties of the $q$-shifting operator ${ }_{a} \Phi_{q}(m)=q m+(1-q) a$ as follows:

(i) ${ }_{a} \Phi_{q}^{k}(m)={ }_{a} \Phi_{q}^{k-1}\left({ }_{a} \Phi_{q}(m)\right)$ with ${ }_{a} \Phi_{q}^{0}(m)=m$, for any positive integer $k$.

(ii) ${ }_{a}(n-m)_{q}^{(0)}=1,{ }_{a}(n-m)_{q}^{(k)}=\prod_{i=0}^{k-1}\left(n-{ }_{a} \Phi_{q}^{i}(m)\right), k \in \mathbb{N} \cup\{\infty\}$.

(iii) ${ }_{a}(n-m)_{q}^{(\gamma)}=n^{(\gamma)} \prod_{i=0}^{\infty} \frac{1-\frac{a}{n} \Phi_{q}^{i}(m / n)}{1-\frac{a}{n} \Phi_{q}^{\gamma+i}(m / n)}, \gamma \in \mathbb{R}$.

Next we turn to the definitions of the Riemann-Liouville fractional $q$-derivative and the $q$-integral on the interval $[a, b]$.

Definition 2.1 [18] The fractional $q$-derivative of Riemann-Liouville type of order $v \geq 0$ on the interval $[a, b]$ is defined by $\left({ }_{a} D_{q}^{0} f\right)(t)=f(t)$ and

$$
\left({ }_{a} D_{q}^{v} f\right)(t)=\left({ }_{a} D_{q^{a}}^{l} I_{q}^{l-v} f\right)(t), \quad v>0
$$

where $l$ is the smallest integer greater than or equal to $v$.

Definition 2.2 [18] Let $\alpha \geq 0$ and $f$ be a function defined on $[a, b]$. The fractional $q$-integral of Riemann-Liouville type is given by $\left({ }_{a} I_{q}^{0} f\right)(t)=f(t)$ and

$$
\left({ }_{a} I_{q}^{\alpha} f\right)(t)=\frac{1}{\Gamma_{q}(\alpha)} \int_{a}^{t}{ }_{a}\left(t-{ }_{a} \Phi_{q}(s)\right)_{q}^{(\alpha-1)} f(s){ }_{a} d_{q} s, \quad \alpha>0, t \in[a, b] .
$$

From [18], we have the following formulas:

$$
\begin{aligned}
& { }_{a} D_{q}^{\alpha}(t-a)^{\beta}=\frac{\Gamma_{q}(\beta+1)}{\Gamma_{q}(\beta-\alpha+1)}(t-a)^{\beta-\alpha}, \\
& { }_{a} I_{q}^{\alpha}(t-a)^{\beta}=\frac{\Gamma_{q}(\beta+1)}{\Gamma_{q}(\beta+\alpha+1)}(t-a)^{\beta+\alpha} .
\end{aligned}
$$

Lemma 2.1 [18] Let $\alpha, \beta \in \mathbb{R}^{+}$and $f$ be a continuous function on $[a, b], a \geq 0$. The Riemann-Liouville fractional q-integral has the following semi-group property:

$$
{ }_{a} I_{q}^{\beta} a I_{q}^{\alpha} f(t)={ }_{a} I_{q}^{\alpha} I_{q}^{\beta} f(t)={ }_{a} I_{q}^{\alpha+\beta} f(t)
$$

Lemma 2.2 [18] Let $f$ be a q-integrable function on $[a, b]$. Then the following equality holds:

$$
{ }_{a} D_{q}^{\alpha} I_{q}^{\alpha} f(t)=f(t), \quad \text { for } \alpha>0, t \in[a, b] .
$$

Lemma 2.3 [18] Let $\alpha>0$ and $p$ be a positive integer. Then for $t \in[a, b]$ the following equality holds:

$$
{ }_{a} I_{q}^{\alpha}{ }_{a} D_{q}^{p} f(t)={ }_{a} D_{q a}^{p} I_{q}^{\alpha} f(t)-\sum_{k=0}^{p-1} \frac{(t-a)^{\alpha-p+k}}{\Gamma_{q}(\alpha+k-p+1)}{ }_{a} D_{q}^{k} f(a) .
$$


In this paper we first give the definition of Caputo fractional $q$-derivative as follows.

Definition 2.3 The fractional $q$-derivative of Caputo type of order $\alpha \geq 0$ on the interval $[a, b]$ is defined by $\left({ }_{a}^{c} D_{q}^{0} f\right)(t)=f(t)$ and

$$
\left({ }_{a}^{c} D_{q}^{\alpha} f\right)(t)=\left({ }_{a} I_{q}^{n-\alpha}{ }_{a} D_{q}^{n} f\right)(t), \quad \alpha>0,
$$

where $n$ is the smallest integer greater than or equal to $\alpha$.

Lemma 2.4 Let $\alpha>0$ and $n$ be the smallest integer greater than or equal to $\alpha$. Then for $t \in[a, b]$, the following equality holds:

$$
{ }_{a} I_{q a}^{\alpha c} D_{q}^{\alpha} f(t)=f(t)-\sum_{k=0}^{n-1} \frac{(t-a)^{k}}{\Gamma_{q}(k+1)}{ }_{a} D_{q}^{k} f(a)
$$

Proof From Lemma 2.3, for $\alpha=p=m$, where $m$ is a positive integer, we have

$$
{ }_{a} I_{q a}^{m} D_{q}^{m} f(t)={ }_{a} D_{q}^{m} a I_{q}^{m} f(t)-\sum_{k=0}^{m-1} \frac{(t-a)^{k}}{\Gamma_{q}(k+1)}{ }_{a} D_{q}^{k} f(a)=f(t)-\sum_{k=0}^{m-1} \frac{(t-a)^{k}}{\Gamma_{q}(k+1)}{ }_{a} D_{q}^{k} f(a) .
$$

Then by Definition 2.3, we have

$$
{ }_{a} I_{q a}^{\alpha c} D_{q}^{\alpha} f(t)={ }_{a} I_{q}^{\alpha} a I_{q}^{n-\alpha}{ }_{a} D_{q}^{n} f(t)={ }_{a} I_{q a}^{n} D_{q}^{n} f(t)=f(t)-\sum_{k=0}^{n-1} \frac{(t-a)^{k}}{\Gamma_{q}(k+1)}{ }_{a} D_{q}^{k} f(a) .
$$

Lemma 2.5 Let $h \in C(J, \mathbb{R})$. Then the unique solution of

$$
\left\{\begin{array}{l}
{ }_{t_{k}}^{c} D_{q_{k}}^{\alpha_{k}} x(t)=h(t), \quad t \in J_{k} \subseteq[0, T], t \neq t_{k}, \\
\Delta x\left(t_{k}\right)=\varphi_{k}\left(t_{k-1} I_{q_{k-1}}^{\beta_{k-1}} x\left(t_{k}\right)\right), \quad k=1,2, \ldots, m, \\
t_{k} D_{q_{k}} x\left(t_{k}^{+}\right)-t_{k-1} D_{q_{k-1}} x\left(t_{k}\right)=\varphi_{k}^{*}\left(t_{k-1} I_{q_{k-1}}^{\gamma_{k-1}} x\left(t_{k}\right)\right), \quad k=1,2, \ldots, m, \\
x(0)=-x(T), \quad{ }_{0} D_{q_{0}} x(0)=-{ }_{t_{m}} D_{q_{m}} x(T),
\end{array}\right.
$$

is given by

$$
\begin{aligned}
x(t)= & -\frac{1}{2} \sum_{i=1}^{m}\left[t_{i-1} I_{q_{i-1}}^{\alpha_{i-1}} h\left(t_{i}\right)+\varphi_{i}\left(t_{i-1} I_{q_{i-1}}^{\beta_{i-1}} x\left(t_{i}\right)\right)\right] \\
& -\frac{1}{2} \sum_{i=1}^{m}\left(T-t_{i}\right)\left\{t_{i-1} I_{q_{i-1}}^{\alpha_{i-1}-1} h\left(t_{i}\right)+\varphi_{i}^{*}\left(t_{i-1} I_{q_{i-1}}^{\gamma_{i-1}} x\left(t_{i}\right)\right)\right\} \\
& -\frac{1}{2} t_{m} I_{q_{m}}^{\alpha_{m}} h(T)+\left(t-\frac{T}{2}\right)\left[-\frac{1}{2} \sum_{i=1}^{m}\left\{t_{i-1} I_{q_{i-1}}^{\alpha_{i-1}-1} h\left(t_{i}\right)\right.\right. \\
& \left.\left.+\varphi_{i}^{*}\left({ }_{t_{i-1}}{ }_{q_{i-1}}^{\gamma_{i-1}} x\left(t_{i}\right)\right)\right\}-\frac{1}{2} t_{m} I_{q_{m}}^{\alpha_{m}-1} h(T)\right] \\
& +\sum_{i=1}^{k}\left[t_{i-1} I_{q_{i-1}}^{\alpha_{i-1}} h\left(t_{i}\right)+\varphi_{i}\left(t_{i-1} I_{q_{i-1}}^{\beta_{i-1}} x\left(t_{i}\right)\right)\right]
\end{aligned}
$$




$$
\begin{aligned}
& +\sum_{i=1}^{k}\left(t-t_{i}\right)\left\{t_{i-1} I_{q_{i-1}}^{\alpha_{i-1}-1} h\left(t_{i}\right)+\varphi_{i}^{*}\left({ }_{t_{i-1}} I_{q_{i-1}}^{\gamma_{i-1}} x\left(t_{i}\right)\right)\right\} \\
& +{ }_{t_{k}} I_{q_{k}}^{\alpha_{k}} h(t),
\end{aligned}
$$

where $\sum_{1}^{0}(\cdot)=0$.

Proof Applying the Riemann-Liouville fractional $q_{0}$-integral operator of order $\alpha_{0}$ on both sides of the first equation of (2.18) for $t \in J_{0}$ and using Lemma 2.4, we obtain

$$
{ }_{t_{0}} I_{q_{0} t_{0}}^{\alpha_{0} c} D_{q_{0}}^{\alpha_{0}} x(t)=x(t)-x(0)-\frac{{ }_{0} D_{q_{0}} x(0)}{\Gamma_{q_{0}}(2)} t={ }_{t_{0}} I_{q_{0}}^{\alpha_{0}} h(t)
$$

which yields

$$
x(t)=C_{0}+C_{1} t+{ }_{t_{0}} I_{q_{0}}^{\alpha_{0}} h(t)
$$

where $C_{0}=x(0)$ and $C_{1}={ }_{0} D_{q_{0}} x(0)$. In particular, for $t=t_{1}$, we have

$$
x\left(t_{1}\right)=C_{0}+C_{1} t_{1}+{ }_{t_{0}} I_{q_{0}}^{\alpha_{0}} h\left(t_{1}\right) \text { and } t_{0} D_{q_{0}} x\left(t_{1}\right)=C_{1}+{ }_{t_{0}} I_{q_{0}}^{\alpha_{0}-1} h\left(t_{1}\right) .
$$

For $t \in J_{1}$, on application of the Riemann-Liouville fractional $q_{1}$-integral operator of order $\alpha_{1}$ to (2.18) and using the above arguments, we get

$$
x(t)=x\left(t_{1}^{+}\right)+\left(t-t_{1}\right)_{t_{1}} D_{q_{1}} x\left(t_{1}^{+}\right)+{ }_{t_{1}} I_{q_{1}}^{\alpha_{1}} h(t) .
$$

Using the impulsive conditions $x\left(t_{1}^{+}\right)=x\left(t_{1}\right)+\varphi_{1}\left(t_{t_{0}} I_{q_{0}}^{\beta_{0}} x\left(t_{1}\right)\right)$ and ${ }_{t_{1}} D_{q_{1}} x\left(t_{1}^{+}\right)={ }_{t_{0}} D_{q_{0}} x\left(t_{1}\right)+$ $\varphi_{1}^{*}\left(t_{0} I_{q_{0}}^{\gamma_{0}} x\left(t_{1}\right)\right)$, we obtain

$$
\begin{aligned}
x(t)= & C_{0}+C_{1} t+\left[t_{0} I_{q_{0}}^{\alpha_{0}} h\left(t_{1}\right)+\varphi_{1}\left(\left(_{t_{0}} I_{q_{0}}^{\beta_{0}} x\left(t_{1}\right)\right)\right]\right. \\
& +\left(t-t_{1}\right)\left[t_{0} I_{q_{0}}^{\alpha_{0}-1} h\left(t_{1}\right)+\varphi_{1}^{*}\left({ }_{t_{0}} I_{q_{0}}^{\gamma_{0}} x\left(t_{1}\right)\right)\right]+{ }_{t_{1}} I_{q_{1}}^{\alpha_{1}} h(t) .
\end{aligned}
$$

In a similar manner, for $t \in J_{2}$, we have

$$
\begin{aligned}
x(t)= & C_{0}+C_{1} t+\left[{ }_{t_{0}} I_{q_{0}}^{\alpha_{0}} h\left(t_{1}\right)+\varphi_{1}\left(t_{t_{0}} I_{q_{0}}^{\beta_{0}} x\left(t_{1}\right)\right)\right]+\left[{ }_{t_{1}} I_{q_{1}}^{\alpha_{1}} h\left(t_{2}\right)+\varphi_{2}\left({ }_{t_{1}} I_{q_{1}}^{\beta_{1}} x\left(t_{2}\right)\right)\right] \\
& +\left(t-t_{1}\right)\left[{ }_{t_{0}} I_{q_{0}}^{\alpha_{0}-1} h\left(t_{1}\right)+\varphi_{1}^{*}\left({ }_{t_{0}} I_{q_{0}}^{\gamma_{0}} x\left(t_{1}\right)\right)\right] \\
& +\left(t-t_{2}\right)\left[{ }_{t_{1}} I_{q_{1}}^{\alpha_{1}-1} h\left(t_{2}\right)+\varphi_{2}^{*}\left({ }_{t_{1}} I_{q_{1}}^{\gamma_{1}} x\left(t_{2}\right)\right)\right]+{ }_{t_{2}} I_{q_{2}}^{\alpha_{2}} h(t) .
\end{aligned}
$$

Repeating the above process, for $t \in J_{k} \subseteq J, k=0,1,2, \ldots, m$, we obtain

$$
\begin{aligned}
x(t)= & C_{0}+C_{1} t+\sum_{i=1}^{k}\left[t_{i-1} I_{q_{i-1} \alpha_{i-1}}^{\alpha_{i}} h\left(t_{i}\right)+\varphi_{i}\left(t_{i-1} I_{q_{i-1}}^{\beta_{i-1}} x\left(t_{i}\right)\right)\right] \\
& +\sum_{i=1}^{k}\left(t-t_{i}\right)\left\{t_{i-1} I_{q_{i-1}}^{\alpha_{i-1}-1} h\left(t_{i}\right)+\varphi_{i}^{*}\left(\left(_{i-1} I_{q_{i-1}}^{\gamma_{i-1}} x\left(t_{i}\right)\right)\right\}\right. \\
& +t_{k} I_{q_{k}}^{\alpha_{k}} h(t),
\end{aligned}
$$


where $\sum_{1}^{0}(\cdot)=0$. Notice that $x(0)=C_{0}$ and

$$
\begin{aligned}
x(T)= & C_{0}+C_{1} T+\sum_{i=1}^{m}\left[t_{i-1} I_{q_{i-1}}^{\alpha_{i-1}} h\left(t_{i}\right)+\varphi_{i}\left(t_{i-1} I_{q_{i-1}}^{\beta_{i-1}} x\left(t_{i}\right)\right)\right] \\
& +\sum_{i=1}^{m}\left(T-t_{i}\right)\left\{t_{i-1} I_{q_{i-1}^{\alpha_{i-1}-1}} h\left(t_{i}\right)+\varphi_{i}^{*}\left(t_{i-1} I_{q_{i-1}}^{\gamma_{i-1}} x\left(t_{i}\right)\right)\right\} \\
& +{ }_{t_{m}} I_{q_{m}}^{\alpha_{m}} h(T) .
\end{aligned}
$$

On the other hand, we have

$$
t_{k} D_{q_{k}} x(t)=C_{1}+\sum_{i=1}^{k}\left\{t_{i-1} I_{q_{i-1}}^{\alpha_{i-1}-1} h\left(t_{i}\right)+\varphi_{i}^{*}\left(t_{i-1}{ }_{q_{i-1}}^{\gamma_{i-1}} x\left(t_{i}\right)\right)\right\}+{ }_{t_{k}} I_{q_{k}}^{\alpha_{k}-1} h(t)
$$

which implies $t_{0} D_{q_{0}} x(0)=C_{1}$ and

$$
{ }_{t_{m}} D_{q_{m}} x(T)=C_{1}+\sum_{i=1}^{m}\left\{t_{i-1} I_{q_{i-1}}^{\alpha_{i-1}-1} h\left(t_{i}\right)+\varphi_{i}^{*}\left(t_{i-1} I_{q_{i-1}}^{\gamma_{i-1}} x\left(t_{i}\right)\right)\right\}+{ }_{t_{m}} I_{q_{m}}^{\alpha_{m}-1} h(T) .
$$

Now making use of the boundary conditions given by (2.18), we find that

$$
\begin{aligned}
C_{0}= & -\frac{1}{2} C_{1} T-\frac{1}{2} \sum_{i=1}^{m}\left[t_{i-1} I_{q_{i-1}}^{\alpha_{i-1}} h\left(t_{i}\right)+\varphi_{i}\left(t_{i-1} I_{q_{i-1}}^{\beta_{i-1}} x\left(t_{i}\right)\right)\right] \\
& -\frac{1}{2} \sum_{i=1}^{m}\left(T-t_{i}\right)\left\{t_{i-1} I_{q_{i-1}^{\alpha_{i-1}-1}}^{\alpha_{1}} h\left(t_{i}\right)+\varphi_{i}^{*}\left(t_{i-1} I_{q_{i-1}}^{\gamma_{i-1}} x\left(t_{i}\right)\right)\right\}-\frac{1}{2} t_{m} I_{q_{m}}^{\alpha_{m}} h(T)
\end{aligned}
$$

and

$$
C_{1}=-\frac{1}{2} \sum_{i=1}^{m}\left\{t_{i-1} I_{q_{i-1}}^{\alpha_{i-1}-1} h\left(t_{i}\right)+\varphi_{i}^{*}\left(t_{i-1} I_{q_{i-1}}^{\gamma_{i-1}} x\left(t_{i}\right)\right)\right\}-\frac{1}{2} t_{m} I_{q_{m}}^{\alpha_{m}-1} h(T) .
$$

Substituting the values $C_{0}$ and $C_{1}$ in (2.23) yields the solution (2.19).

\section{Main results}

Let $P C(J, \mathbb{R})=\left\{x: J \rightarrow \mathbb{R}: x(t)\right.$ is continuous everywhere except for some $t_{k}$ at which $x\left(t_{k}^{+}\right)$ and $x\left(t_{k}^{-}\right)$exist and $\left.x\left(t_{k}^{-}\right)=x\left(t_{k}\right), k=1,2, \ldots, m\right\}$. Observe that $P C(J, \mathbb{R})$ is a Banach space equipped with the norm $\|x\|_{P C}=\sup \{|x(t)|: t \in J\}$.

In view of Lemma 2.5 , we define an operator $\mathcal{A}: P C(J, \mathbb{R}) \rightarrow P C(J, \mathbb{R})$ by

$$
\begin{aligned}
\mathcal{A} x(t)= & -\frac{1}{2} \sum_{i=1}^{m}\left[t_{i-1} I_{q_{i-1}^{\alpha_{i-1}}} f\left(t_{i}, x\left(t_{i}\right)\right)+\varphi_{i}\left(\left(_{i-1} I_{q_{i-1}}^{\beta_{i-1}} x\left(t_{i}\right)\right)\right]\right. \\
& -\frac{1}{2} \sum_{i=1}^{m}\left(T-t_{i}\right)\left\{t_{i-1} I_{q_{i-1}^{\alpha_{i-1}-1}} f\left(t_{i}, x\left(t_{i}\right)\right)+\varphi_{i}^{*}\left({ }_{t_{i-1}} I_{q_{i-1}^{i-1}}^{\gamma_{i-1}} x\left(t_{i}\right)\right)\right\} \\
& -\frac{1}{2} t_{m} I_{q_{m}}^{\alpha_{m}} f(T, x(T))+\left(t-\frac{T}{2}\right)\left[-\frac{1}{2} \sum_{i=1}^{m}\left\{t_{i_{i-1}} I_{q_{i-1}}^{\alpha_{i-1}-1} f\left(t_{i}, x\left(t_{i}\right)\right)\right.\right.
\end{aligned}
$$




$$
\begin{aligned}
& \left.\left.+\varphi_{i}^{*}\left(t_{i-1} I_{q_{i-1}}^{\gamma_{i-1}} x\left(t_{i}\right)\right)\right\}-\frac{1}{2} t_{m} I_{q_{m}}^{\alpha_{m}-1} f(T, x(T))\right] \\
& +\sum_{i=1}^{k}\left[t_{i-1} I_{q_{i-1}}^{\alpha_{i-1}} f\left(t_{i}, x\left(t_{i}\right)\right)+\varphi_{i}\left(t_{i-1} I_{q_{i-1}}^{\beta_{i-1}} x\left(t_{i}\right)\right)\right] \\
& +\sum_{i=1}^{k}\left(t-t_{i}\right)\left\{t_{i-1} I_{q_{i-1}}^{\alpha_{i-1}-1} f\left(t_{i}, x\left(t_{i}\right)\right)+\varphi_{i}^{*}\left({ }_{t_{i-1}} I_{q_{i-1}}^{\gamma_{i-1}} x\left(t_{i}\right)\right)\right\} \\
& +t_{k} I_{q_{k}}^{\alpha_{k}} f(t, x(t)),
\end{aligned}
$$

where

$$
{ }_{a} I_{q}^{p} f(u, x(u))=\frac{1}{\Gamma_{q}(p)} \int_{a}^{u}{ }_{a}\left(u-{ }_{a} \Phi_{q}(s)\right)_{q}^{(p-1)} f(s, x(s)){ }_{a} d_{q} s,
$$

$p \in\left\{\alpha_{0}, \ldots, \alpha_{m}, \alpha_{0}-1, \ldots, \alpha_{m}-1, \beta_{0}, \ldots, \beta_{m-1}, \gamma_{0}, \ldots, \gamma_{m-1}\right\}, q \in\left\{q_{0}, \ldots, q_{m}\right\}, a \in\left\{t_{0}, \ldots, t_{m}\right\}$, and $u \in\left\{t, t_{1}, t_{2}, \ldots, t_{m}, T\right\}$.

For computational convenience, we set

$$
\begin{aligned}
\Omega_{1}= & \frac{3}{2} \sum_{i=1}^{m+1} \frac{\left(t_{i}-t_{i-1}\right)^{\alpha_{i-1}}}{\Gamma_{q_{i-1}}\left(\alpha_{i-1}+1\right)}+\frac{3}{2} \sum_{i=1}^{m} \frac{\left(T-t_{i}\right)\left(t_{i}-t_{i-1}\right)^{\alpha_{i-1}-1}}{\Gamma_{q_{i-1}}\left(\alpha_{i-1}\right)} \\
& +\frac{T}{4} \sum_{i=1}^{m+1} \frac{\left(t_{i}-t_{i-1}\right)^{\alpha_{i-1}-1}}{\Gamma_{q_{i-1}}\left(\alpha_{i-1}\right)} \\
\Omega_{2}= & \frac{3}{2} m M_{1}+\frac{3}{2} M_{2} \sum_{i=1}^{m}\left(T-t_{i}\right)+\frac{T}{4} m M_{2} .
\end{aligned}
$$

Now we present our first existence result for the problem (1.1), which is based on the Schauder fixed point theorem.

\section{Theorem 3.1 Assume that}

$\left(\mathrm{H}_{1}\right)$ there exist continuous functions $a(t), b(t)$, and nonnegative constants $M_{1}, M_{2}$ such that

$$
|f(t, x)| \leq a(t)+b(t)|x|, \quad(t, x) \in J \times \mathbb{R}
$$

with $\sup _{t \in J}|a(t)|=a_{1}, \sup _{t \in J}|b(t)|=b_{1}$, and

$$
\left|\varphi_{k}(x)\right| \leq M_{1}, \quad\left|\varphi_{k}^{*}(x)\right| \leq M_{2}, \quad \forall x \in \mathbb{R}, k=1,2, \ldots, m
$$

Then the anti-periodic boundary value problem (1.1) has at least one solution on $J$ if

$$
b_{1} \Omega_{1}<1 \text {. }
$$

Proof Let us define a closed ball $B_{R}=\left\{x \in P C(J, \mathbb{R}):\|x\|_{P C} \leq R\right\}$ with

$$
R>\frac{a_{1} \Omega_{1}+\Omega_{2}}{1-b_{1} \Omega_{1}}
$$


where $a_{1}, b_{1}$ are defined in $\left(\mathrm{H}_{1}\right)$ and $\Omega_{1}, \Omega_{2}$ are, respectively, given by (3.2) and (3.3). Clearly $B_{R}$ is a bounded, closed, and convex subset of $P C(J, \mathbb{R})$. Now we show that the operator $\mathcal{A}: P C(J, \mathbb{R}) \rightarrow P C(J, \mathbb{R})$ defined by (3.1) has a fixed point in the following two steps.

Step 1. $\mathcal{A}: B_{R} \rightarrow B_{R}$.

For any $x \in B_{R}$, using (2.12), we have

$$
\begin{aligned}
& |\mathcal{A} x(t)| \leq \frac{1}{2} \sum_{i=1}^{m}\left[t_{i-1} I_{q_{i-1}}^{\alpha_{i-1}}\left|f\left(t_{i}, x\left(t_{i}\right)\right)\right|+\left|\varphi_{i}\left(t_{i-1} I_{q_{i-1}}^{\beta_{i-1}} x\left(t_{i}\right)\right)\right|\right] \\
& +\frac{1}{2} \sum_{i=1}^{m}\left(T-t_{i}\right)\left\{t_{i-1} I_{q_{i-1}}^{\alpha_{i-1}-1}\left|f\left(t_{i}, x\left(t_{i}\right)\right)\right|+\left|\varphi_{i}^{*}\left(t_{i-1}{ }_{q_{i-1}}^{\gamma_{i-1}} x\left(t_{i}\right)\right)\right|\right\} \\
& +\frac{1}{2} t_{m} I_{q_{m}}^{\alpha_{m}}|f(T, x(T))|+\frac{T}{2}\left[\frac { 1 } { 2 } \sum _ { i = 1 } ^ { m } \left\{{ }_{t_{i-1}} I_{q_{i-1}}^{\alpha_{i-1}-1}\left|f\left(t_{i}, x\left(t_{i}\right)\right)\right|\right.\right. \\
& \left.+\left|\varphi_{i}^{*}\left(\left(_{i-1} I_{q_{i-1}}^{\gamma_{i-1}} x\left(t_{i}\right)\right) \mid\right\}+\frac{1}{2} t_{m} I_{q_{m}}^{\alpha_{m}-1}\right| f(T, x(T)) \mid\right] \\
& +\sum_{i=1}^{k}\left[t_{i-1} I_{q_{i-1}}^{\alpha_{i-1}}\left|f\left(t_{i}, x\left(t_{i}\right)\right)\right|+\left|\varphi_{i}\left(t_{i-1} I_{q_{i-1}}^{\beta_{i-1}} x\left(t_{i}\right)\right)\right|\right] \\
& +\sum_{i=1}^{k}\left(t-t_{i}\right)\left\{t_{i-1} I_{q_{i-1}}^{\alpha_{i-1}-1}\left|f\left(t_{i}, x\left(t_{i}\right)\right)\right|+\left|\varphi_{i}^{*}\left(t_{i-1} I_{q_{i-1}}^{\gamma_{i-1}} x\left(t_{i}\right)\right)\right|\right\} \\
& +{ }_{t_{k}} I_{q_{k}}^{\alpha_{k}}|f(t, x(t))| \\
& \leq \frac{1}{2} \sum_{i=1}^{m}\left[\left(a_{1}+b_{1}\|x\|_{P C}\right) t_{i-1} I_{q_{i-1}}^{\alpha_{i-1}} 1\left(t_{i}\right)+M_{1}\right] \\
& +\frac{1}{2} \sum_{i=1}^{m}\left(T-t_{i}\right)\left\{\left(a_{1}+b_{1}\|x\|_{P C}\right)_{t_{i-1}} I_{q_{i-1}}^{\alpha_{i-1}-1} 1\left(t_{i}\right)+M_{2}\right\} \\
& +\frac{1}{2}\left(a_{1}+b_{1}\|x\|_{P C}\right)_{t_{m}} I_{q_{m}}^{\alpha_{m}} 1(T) \\
& +\frac{T}{2}\left[\frac{1}{2} \sum_{i=1}^{m}\left\{\left(a_{1}+b_{1}\|x\|_{P C}\right)_{t_{i-1}} I_{q_{i-1}}^{\alpha_{i-1}-1} 1\left(t_{i}\right)+M_{2}\right\}\right. \\
& \left.+\frac{1}{2}\left(a_{1}+b_{1}\|x\|_{P C}\right)_{t_{m}} I_{q_{m}}^{\alpha_{m}-1} 1(T)\right] \\
& +\sum_{i=1}^{m}\left[\left(a_{1}+b_{1}\|x\|_{P C}\right)_{t_{i-1}} I_{q_{i-1}}^{\alpha_{i-1}} 1\left(t_{i}\right)+M_{1}\right] \\
& +\sum_{i=1}^{m}\left(T-t_{i}\right)\left\{\left(a_{1}+b_{1}\|x\|_{P C}\right)_{t_{i-1}} I_{q_{i-1}}^{\alpha_{i-1}-1} 1\left(t_{i}\right)+M_{2}\right\} \\
& +\left(a_{1}+b_{1}\|x\|_{P C}\right)_{t_{m}} I_{q_{m}}^{\alpha_{m}} 1(T) \\
& =\frac{3}{2} \sum_{i=1}^{m+1} \frac{\left(t_{i}-t_{i-1}\right)^{\alpha_{i-1}}}{\Gamma_{q_{i-1}}\left(\alpha_{i-1}+1\right)}\left(a_{1}+b_{1}\|x\|_{P C}\right)+\frac{3}{2} m M_{1} \\
& +\frac{3}{2} \sum_{i=1}^{m}\left(T-t_{i}\right)\left\{\frac{\left(t_{i}-t_{i-1}\right)^{\alpha_{i-1}-1}}{\Gamma_{q_{i-1}}\left(\alpha_{i-1}\right)}\left(a_{1}+b_{1}\|x\|_{P C}\right)+M_{2}\right\}
\end{aligned}
$$




$$
\begin{aligned}
& +\frac{T}{4} \sum_{i=1}^{m+1} \frac{\left(t_{i}-t_{i-1}\right)^{\alpha_{i-1}-1}}{\Gamma_{q_{i-1}}\left(\alpha_{i-1}\right)}\left(a_{1}+b_{1}\|x\|_{P C}\right)+\frac{T}{4} M_{2} m \\
= & a_{1} \Omega_{1}+\Omega_{2}+b_{1}\|x\|_{P C} \Omega_{1} \leq R
\end{aligned}
$$

which implies $\|\mathcal{A} x\|_{P C} \leq R$. Therefore, $\mathcal{A}: B_{R} \rightarrow B_{R}$.

Step 2. The operator $\mathcal{A}: P C(J, \mathbb{R}) \rightarrow P C(J, \mathbb{R})$ is completely continuous on $B_{R}$.

Let $\sup _{(t, x) \in J \times B_{R}}|f(t, x)|=F_{1}$. For any $\tau_{1}, \tau_{2} \in J_{k}, k=0,1, \ldots, m$, with $\tau_{1}<\tau_{2}$, we have

$$
\begin{aligned}
\left|\mathcal{A} x\left(\tau_{2}\right)-\mathcal{A} x\left(\tau_{1}\right)\right| \leq & \left|\tau_{2}-\tau_{1}\right|\left[\frac{1}{2} \sum_{i=1}^{m+1} \frac{\left(t_{i}-t_{i-1}\right)^{\alpha_{i-1}-1}}{\Gamma_{q_{i-1}}\left(\alpha_{i-1}\right)} F_{1}+\frac{m M_{2}}{2}\right] \\
& +\left|\tau_{2}-\tau_{1}\right| \sum_{i=1}^{k}\left[\frac{\left(t_{i}-t_{i-1}\right)^{\alpha_{i-1}-1}}{\Gamma_{q_{i-1}}\left(\alpha_{i-1}\right)} F_{1}+M_{2}\right] \\
& +\frac{F_{1}}{\Gamma_{q_{k}}\left(\alpha_{k}\right)} \mid \int_{t_{k}}^{\tau_{2}} t_{k}\left(\tau_{2}-t_{k} \Phi_{q_{k}}\right)_{q_{k}}^{\left(\alpha_{k}-1\right)}{ }_{t_{k}} d_{q_{k}} s \\
& -\int_{t_{k}}^{\tau_{1}} t_{k}\left(\tau_{1}-t_{k} \Phi_{q_{k}}\right)_{q_{k}}^{\left(\alpha_{k}-1\right)} t_{t_{k}} d_{q_{k}} s \mid,
\end{aligned}
$$

which is independent of $x$ and tends to zero as $\tau_{2}-\tau_{1} \rightarrow 0$. Therefore $\mathcal{A}$ is equicontinuous. Thus $\mathcal{A} B_{R}$ is relatively compact as $\mathcal{A} B_{R} \subset B_{R}$ is uniformly bounded. In view of the continuity of $f, \varphi_{k}$ and $\varphi_{k}^{*}, k=1,2, \ldots, m$, it is clear that the operator $\mathcal{A}$ is continuous. Hence the operator $\mathcal{A}: P C(J, \mathbb{R}) \rightarrow P C(J, \mathbb{R})$ is completely continuous on $B_{R}$. Applying the Schauder fixed point theorem, we deduce that the operator $\mathcal{A}$ has at least one fixed point in $B_{R}$. This shows that the problem (1.1) has at least one solution on $J$.

In the next existence result, we make use of Leray-Schauder's nonlinear alternative.

Lemma 3.1 (Nonlinear alternative for single valued maps) [19] Let E be a Banach space, $C$ a closed, convex subset of $E, U$ an open subset of $C$, and $0 \in U$. Suppose that $F: \bar{U} \rightarrow C$ is a continuous, compact (that is, $F(\bar{U})$ is a relatively compact subset of $C$ ) map. Then either

(i) F has a fixed point in $\bar{U}$, or

(ii) there is a $u \in \partial U$ (the boundary of $U$ in $C$ ) and $\theta \in(0,1)$ with $u=\theta F(u)$.

In the sequel, we set

$$
\begin{aligned}
& \Omega_{3}=\frac{3}{2} \sum_{i=1}^{m} \frac{\left(t_{i}-t_{i-1}\right)^{\beta_{i-1}}}{\Gamma_{q_{i-1}}\left(\beta_{i-1}+1\right)}, \\
& \Omega_{4}=\frac{3}{2} \sum_{i=1}^{m} \frac{\left(T-t_{i}\right)\left(t_{i}-t_{i-1}\right)^{\gamma_{i-1}}}{\Gamma_{q_{i-1}}\left(\gamma_{i-1}+1\right)}+\frac{T}{4} \sum_{i=1}^{m} \frac{\left(t_{i}-t_{i-1}\right)^{\gamma_{i-1}}}{\Gamma_{q_{i-1}}\left(\gamma_{i-1}+1\right)} .
\end{aligned}
$$

\section{Theorem 3.2 Assume that}

$\left(\mathrm{H}_{2}\right)$ there exist a continuous nondecreasing function $\psi:[0, \infty) \rightarrow(0, \infty)$, a continuous function $p: J \rightarrow \mathbb{R}^{+}$with $p^{*}=\sup _{t \in J}|p(t)|$ and constants $M_{3}, M_{4}>0$ such that

$$
|f(t, x)| \leq p(t) \psi(|x|), \quad \forall(t, x) \in J \times \mathbb{R}
$$


and

$$
\left|\varphi_{k}(x)\right| \leq M_{3}|x|, \quad\left|\varphi_{k}^{*}(x)\right| \leq M_{4}|x|, \quad \forall x \in R, k=1, \ldots, m ;
$$

$\left(\mathrm{H}_{3}\right)$ there exists a constant $N>0$ such that

$$
\frac{\left(1-M_{3} \Omega_{3}-M_{4} \Omega_{4}\right) N}{p^{*} \psi(N) \Omega_{1}}>1, \quad M_{3} \Omega_{3}+M_{4} \Omega_{4}<1
$$

where $\Omega_{3}, \Omega_{4}$ are, respectively, given by (3.7) and (3.8).

Then the problem (1.1) has at least one solution J.

Proof We shall show that the operator $\mathcal{A}$ defined by (2.5) has a fixed point. To accomplish this, for a positive number $\rho$, let $B_{\rho}=\left\{x \in P C(J, \mathbb{R}):\|x\|_{P C} \leq \rho\right\}$ denote a closed ball in $P C(J, \mathbb{R})$. Then for $x \in B_{\rho}, t \in J$, and using (2.12), we have

$$
\begin{aligned}
& |\mathcal{A} x(t)| \leq \frac{1}{2} \sum_{i=1}^{m}\left[p^{*} \psi(\rho)_{t_{i-1}} I_{q_{i-1}^{i-1}}^{\alpha_{i-1}} 1\left(t_{i}\right)+\rho M_{3 t_{i-1}} I_{q_{i-1}}^{\beta_{i-1}} 1\left(t_{i}\right)\right] \\
& +\frac{1}{2} \sum_{i=1}^{m}\left(T-t_{i}\right)\left\{p^{*} \psi(\rho)_{t_{i-1}} I_{q_{i-1}}^{\alpha_{i-1}-1} 1\left(t_{i}\right)+\rho M_{4 t_{i-1}} I_{q_{i-1}}^{\gamma_{i-1}} 1\left(t_{i}\right)\right\} \\
& +\frac{1}{2} p^{*} \psi(\rho)_{t_{m}} I_{q_{m}}^{\alpha_{m}} 1(T)+\frac{T}{2}\left[\frac { 1 } { 2 } \sum _ { i = 1 } ^ { m } \left\{p^{*} \psi(\rho)_{t_{i-1}} I_{q_{i-1}}^{\alpha_{i-1}-1} 1\left(t_{i}\right)\right.\right. \\
& \left.\left.+\rho M_{4 t_{i-1}} I_{q_{i-1}}^{\gamma_{i-1}} 1\left(t_{i}\right)\right\}+\frac{1}{2} p^{*} \psi(\rho)_{t_{m}} I_{q_{m}}^{\alpha_{m}-1} 1(T)\right] \\
& +\sum_{i=1}^{m}\left[p^{*} \psi(\rho)_{t_{i-1}} I_{q_{i-1}}^{\alpha_{i-1}} 1\left(t_{i}\right)+\rho M_{3 t_{i-1}} I_{q_{i-1}}^{\beta_{i-1}} 1\left(t_{i}\right)\right] \\
& +\sum_{i=1}^{m}\left(T-t_{i}\right)\left\{p^{*} \psi(\rho)_{t_{i-1}} I_{q_{i-1}^{i-1}}^{\alpha_{i-1}} 1\left(t_{i}\right)+\rho M_{4 t_{i-1}} I_{q_{i-1}}^{\gamma_{i-1}} 1\left(t_{i}\right)\right\} \\
& +p^{*} \psi(\rho)_{t_{m}} I_{q_{m}}^{\alpha_{m}} 1(T) \\
& =p^{*} \psi(\rho) \Omega_{1}+\rho M_{3} \Omega_{3}+\rho M_{4} \Omega_{4}:=K \text {, }
\end{aligned}
$$

which implies that $\|\mathcal{A} x\|_{P C} \leq K$.

To show that the operator $\mathcal{A}$ maps bounded sets into equicontinuous sets of $P C(J, \mathbb{R})$, we take $\tau_{1}, \tau_{2} \in J_{k}$ for some $k \in\{0,1,2, \ldots, m\}$ with $\tau_{1}<\tau_{2}$ and $x \in B_{\rho}$. Then we have

$$
\begin{aligned}
& \left|\mathcal{A} x\left(\tau_{2}\right)-\mathcal{A} x\left(\tau_{1}\right)\right| \\
& \leq\left|\tau_{2}-\tau_{1}\right|\left[\frac{p^{*} \psi(\rho)}{2} \sum_{i=1}^{m+1} \frac{\left(t_{i}-t_{i-1}\right)^{\alpha_{i-1}-1}}{\Gamma_{q_{i-1}}\left(\alpha_{i-1}\right)}+\frac{\rho M_{4}}{2} \sum_{i=1}^{m} \frac{\left(t_{i}-t_{i-1}\right)^{\gamma_{i-1}}}{\Gamma_{q_{i-1}}\left(\gamma_{i-1}+1\right)}\right] \\
& \quad+\left|\tau_{2}-\tau_{1}\right| \sum_{i=1}^{k}\left[\frac{\left(t_{i}-t_{i-1}\right)^{\alpha_{i-1}-1}}{\Gamma_{q_{i-1}}\left(\alpha_{i-1}\right)} p^{*} \psi(\rho)+\rho M_{4} \frac{\left(t_{i}-t_{i-1}\right)^{\gamma_{i-1}}}{\Gamma_{q_{i-1}}\left(\gamma_{i-1}+1\right)}\right] \\
& \quad+\frac{p^{*} \psi(\rho)}{\Gamma_{q_{k}}\left(\alpha_{k}\right)}\left|\int_{t_{k}}^{\tau_{2}} t_{k}\left(\tau_{2}-t_{k} \Phi_{q_{k}}\right)_{q_{k}}^{\left(\alpha_{k}-1\right)} t_{k} d_{q_{k}} s-\int_{t_{k}}^{\tau_{1}} t_{k}\left(\tau_{1}-t_{k} \Phi_{q_{k}}\right)_{q_{k}}^{\left(\alpha_{k}-1\right)}{ }_{t_{k}} d_{q_{k}} s\right|
\end{aligned}
$$


which tends to zero independent of $x$ as $\tau_{1} \rightarrow \tau_{2}$. Thus, by the Arzelá-Ascoli theorem, the operator $\mathcal{A}: P C(J, \mathbb{R}) \rightarrow P C(J, \mathbb{R})$ is completely continuous.

Finally, for $\lambda \in(0,1)$, let $x=\lambda \mathcal{A} x$. Then, as in the first step, we can get

$$
\|x\|_{P C} \leq p^{*} \psi\left(\|x\|_{P C}\right) \Omega_{1}+\|x\|_{P C} M_{3} \Omega_{3}+\|x\|_{P C} M_{4} \Omega_{4}
$$

which can alternatively be written as

$$
\frac{\left(1-M_{3} \Omega_{3}-M_{4} \Omega_{4}\right)\|x\|_{P C}}{p^{*} \psi\left(\|x\|_{P C}\right) \Omega_{1}} \leq 1 .
$$

In view of $\left(\mathrm{H}_{3}\right)$, there exists $N$ such that $\|x\|_{P C} \neq N$. We define $\mathcal{U}=\left\{x \in P C(J, \mathbb{R}):\|x\|_{P C}<\right.$ $N$ \}. Note that the operator $\mathcal{A}: \overline{\mathcal{U}} \rightarrow P C$ is continuous and completely continuous. From the choice of $\mathcal{U}$, there is no $x \in \partial \mathcal{U}$ such that $x=\lambda \mathcal{A} x$ for some $\lambda \in(0,1)$. Consequently, by the nonlinear alternative of Leray-Schauder type (Lemma 3.1), we deduce that $\mathcal{A}$ has a fixed point $x \in \overline{\mathcal{U}}$ which is a solution of the problem (1.1) on $J$. This completes the proof.

In the last theorem, we apply Banach's contraction principle to establish the uniqueness of solutions for the problem (1.1).

Theorem 3.3 Assume that there exist a function $\mathcal{W}(t) \in C\left(J, \mathbb{R}^{+}\right)$with $W=\sup _{t \in J}|\mathcal{W}(t)|$ and positive constants $M_{5}, M_{6}$ such that

$$
|f(t, x)-f(t, y)| \leq \mathcal{W}(t)|x-y|, \quad \forall(t, x) \in J \times \mathbb{R}
$$

and

$$
\begin{aligned}
& \left|\varphi_{k}(x)-\varphi_{k}(y)\right| \leq M_{5}|x-y|, \\
& \left|\varphi_{k}^{*}(x)-\varphi_{k}^{*}(y)\right| \leq M_{6}|x-y|, \quad x, y \in \mathbb{R},
\end{aligned}
$$

for $k=1,2, \ldots$, m. If

$$
W \Omega_{1}+M_{5} \Omega_{3}+M_{6} \Omega_{4}<1,
$$

then the problem (1.1) has a unique solution on J.

Proof For any $x, y \in P C(J, \mathbb{R})$, we have

$$
\begin{aligned}
\mid \mathcal{A} x(t) & -\mathcal{A} y(t) \mid \\
\leq & \frac{1}{2} \sum_{i=1}^{m}\left[t_{i-1} I_{q_{i-1}}^{\alpha_{i-1}}\left|f\left(t_{i}, x\right)-f\left(t_{i}, y\right)\right|+M_{5 t_{i-1}} I_{q_{i-1}}^{\beta_{i-1}}|x-y|\left(t_{i}\right)\right] \\
& +\frac{1}{2} \sum_{i=1}^{m}\left(T-t_{i}\right)\left\{t_{i-1} I_{q_{i-1}^{\alpha_{i-1}-1}}^{\alpha_{1}}\left|f\left(t_{i}, x\right)-f\left(t_{i}, y\right)\right|+M_{6 t_{i-1}} I_{q_{i-1}}^{\gamma_{i-1}}|x-y|\left(t_{i}\right)\right\} \\
& +\frac{1}{2}{ }_{t_{m}} I_{q_{m}}^{\alpha_{m}}|f(T, x)-f(T, y)|+\frac{T}{2}\left[\frac { 1 } { 2 } \sum _ { i = 1 } ^ { m } \left\{t_{t_{i-1}} I_{q_{i-1}}^{\alpha_{i-1}-1}\left|f\left(t_{i}, x\right)-f\left(t_{i}, y\right)\right|\right.\right.
\end{aligned}
$$




$$
\begin{aligned}
& \left.\left.+M_{6 t_{i-1}} I_{q_{i-1}^{i-1}}^{\gamma_{i-1}}|x-y|\left(t_{i}\right)\right\}+\frac{1}{2} t_{m} I_{q_{m}}^{\alpha_{m}-1}|f(T, x)-f(T, y)|\right] \\
& +\sum_{i=1}^{k}\left[t_{i-1} I_{q_{i-1}}^{\alpha_{i-1}}\left|f\left(t_{i}, x\right)-f\left(t_{i}, y\right)\right|+M_{5 t_{i-1}} I_{q_{i-1}}^{\beta_{i-1}}|x-y|\left(t_{i}\right)\right] \\
& +\sum_{i=1}^{k}\left(t-t_{i}\right)\left\{{ }_{t_{i-1}} I_{q_{i-1}}^{\alpha_{i-1}-1}\left|f\left(t_{i}, x\right)-f\left(t_{i}, y\right)\right|+M_{6 t_{i-1}} I_{q_{i-1}}^{\gamma_{i-1}}|x-y|\left(t_{i}\right)\right\} \\
& +{ }_{t_{k}} I_{q_{k}}^{\alpha_{k}}|f(t, x)-f(t, y)| \\
& \leq\left(W \Omega_{1}+M_{5} \Omega_{3}+M_{6} \Omega_{4}\right)\|x-y\|_{P C},
\end{aligned}
$$

which yields

$$
\|\mathcal{A} x-\mathcal{A} y\|_{P C} \leq\left(W \Omega_{1}+M_{5} \Omega_{3}+M_{6} \Omega_{4}\right)\|x-y\|_{P C} .
$$

By (3.14), we conclude that $\mathcal{A}$ is a contraction. Thus, by Banach's contraction mapping principle, the problem (1.1) has a unique solution on $J$. This completes the proof.

\section{Examples}

In this section, we present three examples to illustrate our results.

Example 4.1 Consider the following anti-periodic boundary value problem for impulsive Caputo fractional $q$-difference equations:

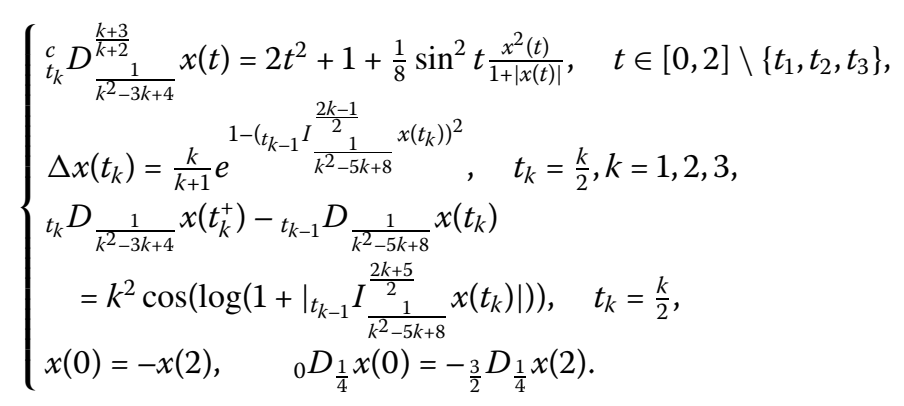

Here $\alpha_{k}=(k+3) /(k+2), q_{k}=1 /\left(k^{2}-3 k+4\right), k=0,1,2,3, \beta_{k-1}=(2 k-1) / 2, \gamma_{k-1}=(2 k+$ $5) / 2, t_{k}=k / 2, k=1,2,3, m=3, T=2$. With the given information, it is found that $\Omega_{1}=$ 7.575532753. Also, we have

$$
\begin{aligned}
& |f(t, x)|=\left|2 t^{2}+1+\frac{1}{8} \sin ^{2} t \frac{x^{2}}{1+|x|}\right| \leq 2 t^{2}+1+\frac{1}{8} \sin ^{2} t|x|, \\
& \left|\varphi_{k}(y)\right|=\left|\frac{k}{k+1} e^{1-y^{2}}\right| \leq e, \quad\left|\varphi_{k}^{*}(z)\right|=\left|k^{2} \cos (\log (1+|z|))\right| \leq 9, \quad k=1,2,3 .
\end{aligned}
$$

With $B=\sup _{t \in[0,2]}\left|(1 / 8) \sin ^{2} t\right|=1 / 8$, we obtain $B \Omega_{1}=0.9469415941<1$. Thus all the conditions of Theorem 3.1 are satisfied. Therefore, by the conclusion of Theorem 3.1, the problem (4.1) has at least one solution on $[0,2]$. 
Example 4.2 Consider the anti-periodic impulsive boundary value problem of fractional $q$-difference equations given by

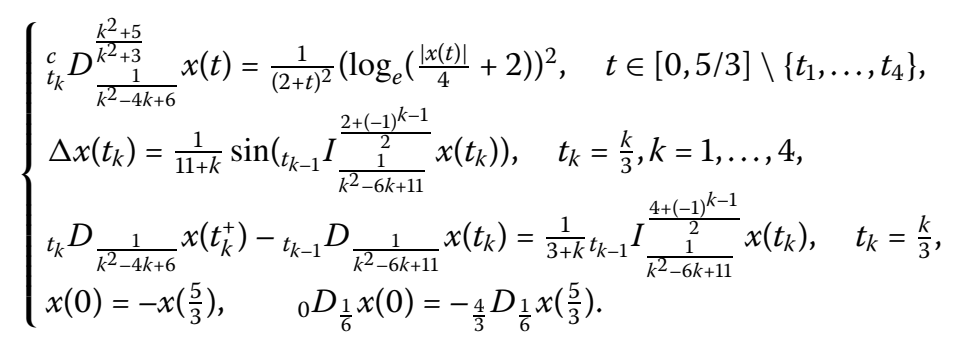

Here $\alpha_{k}=\left(k^{2}+5\right) /\left(k^{2}+3\right), q_{k}=1 /\left(k^{2}-4 k+6\right), k=0,1,2,3,4, \beta_{k-1}=\left(2+(-1)^{k-1}\right) / 2$, $\gamma_{k-1}=\left(4+(-1)^{k-1}\right) / 2, t_{k}=k / 3, k=1,2,3,4, m=4, T=5 / 3$. Using the above data, we find that $\Omega_{1}=6.316994013, \Omega_{3}=2.358729544, \Omega_{4}=0.6481929403$, and

$$
\begin{aligned}
& |f(t, x)|=\left|\frac{1}{(2+t)^{2}}\left(\log _{e}\left(\frac{|x|}{4}+2\right)\right)^{2}\right| \leq \frac{1}{(2+t)^{2}}\left(\frac{|x|}{4}+2\right), \\
& \left|\varphi_{k}(y)\right|=\frac{1}{11+k}|\sin y| \leq \frac{1}{12}|y|, \quad\left|\varphi_{k}^{*}(z)\right|=\frac{1}{3+k}|z| \leq \frac{1}{4}|z|, \quad k=1,2,3,4 .
\end{aligned}
$$

Setting $\psi(x)=(x / 4)+2, p^{*}=\sup _{t \in[0,5 / 3]}\left|1 /(2+t)^{2}\right|=1 / 4, M_{3}=1 / 12$, and $M_{4}=1 / 4$, we find that $M_{3} \Omega_{3}+M_{4} \Omega_{4}=0.3586090304<1$. Also, there exists a constant $N$ such that $N>12.80927819$ satisfying (3.11). Clearly the hypothesis of Theorem 3.2 holds true. Thus the conclusion of Theorem 3.2 implies that the problem (4.2) has at least one solution on $[0,5 / 3]$.

Example 4.3 Consider the following impulsive anti-periodic problem of a fractional $q$-difference equation:

$$
\left\{\begin{array}{l}
{ }_{t_{k}}^{c} D_{\frac{k^{2}+k+3}{k^{2}+2}}^{\frac{1}{k^{2}-5 k+8}} x(t)=\frac{e^{-t} \sin (2 t+1)}{t^{2}+30} \frac{x^{2}(t)+2|x(t)|}{1+|x(t)|}+\frac{1}{2}, \quad t \in[0,3 / 2] \backslash\left\{t_{1}, \ldots, t_{5}\right\}, \\
\Delta x\left(t_{k}\right)=\frac{7 k}{25} \tan ^{-1}\left(t_{k-1} I^{\frac{2 k+1}{2}} \frac{1}{k^{2}-7 k+14} x\left(t_{k}\right)\right)+\frac{2}{3}, \quad t_{k}=\frac{k}{4}, k=1, \ldots, 5, \\
\left.t_{k} D_{\frac{1}{k^{2}-5 k+8}} x\left(t_{k}^{+}\right)-t_{k-1} D_{\frac{1}{k^{2}-7 k+14}} x\left(t_{k}\right)=\frac{\left|t_{k-1} \frac{I^{\frac{k}{2}-4 k+3} \frac{1}{k^{2}-7 k+14}}{k^{2}} x\left(t_{k}\right)\right|}{5 k\left(1+\mid t_{k-1} \frac{2 k^{2}-4 k+3}{2}\right.} x\left(t_{k}\right) \mid\right) \\
x(0)=-x\left(\frac{3}{2}\right), \quad t_{k}=\frac{k}{4}, \\
{ }_{0} D_{\frac{1}{8}} x(0)=-{ }_{\frac{5}{4}} D_{\frac{1}{8}} x\left(\frac{3}{2}\right) .
\end{array}\right.
$$

Here $\alpha_{k}=\left(k^{2}+k+3\right) /\left(k^{2}+2\right), q_{k}=1 /\left(k^{2}-5 k+8\right), k=0,1,2,3,4,5, \beta_{k-1}=(2 k+1) / 2$, $\gamma_{k-1}=\left(2 k^{2}-4 k+3\right) / 2, t_{k}=k / 4, k=1,2,3,4,5, m=5, T=3 / 2$. With the given values, we find that $\Omega_{1}=5.173430458, \Omega_{3}=0.2141916028$, and $\Omega_{4}=1.375385103$. Also, we have

$$
\begin{aligned}
& \left|f\left(t, x_{1}\right)-f\left(t, x_{2}\right)\right| \leq\left|\frac{2 e^{-t} \sin (2 t+1)}{t^{2}+30}\right|\left|x_{1}-x_{2}\right|, \\
& \left|\varphi_{k}\left(y_{1}\right)-\varphi_{k}\left(y_{2}\right)\right|=\frac{7 k}{25}\left|\tan ^{-1} y_{1}-\tan ^{-1} y_{2}\right| \leq \frac{7}{5}\left|y_{1}-y_{2}\right|, \\
& \left|\varphi_{k}^{*}\left(z_{1}\right)-\varphi_{k}^{*}\left(z_{2}\right)\right|=\frac{1}{5 k}\left|\frac{\left|z_{1}\right|}{1+\left|z_{1}\right|}-\frac{\left|z_{2}\right|}{1+\left|z_{2}\right|}\right| \leq \frac{1}{5}\left|z_{1}-z_{2}\right|, \quad k=1,2,3,4,5 .
\end{aligned}
$$


It is easy to see that $W=1 / 15$. Hence, $W \Omega_{1}+M_{5} \Omega_{3}+M_{6} \Omega_{4}=0.9198406284<1$. Thus all the conditions of Theorem 3.3 are satisfied. Hence it follows by the conclusion of Theorem 3.3 that the problem (4.3) has a unique solution on [0,3/2].

\section{Competing interests}

The authors declare that they have no competing interests.

\section{Authors' contributions}

Each of the authors, BA, JT, SKN, HHA, and SM, contributed to each part of this work equally and read and approved the final version of the manuscript.

\section{Author details}

'Nonlinear Analysis and Applied Mathematics (NAAM) Research Group, Department of Mathematics, Faculty of Science, King Abdulaziz University, P.O. Box 80203, Jeddah, 21589, Saudi Arabia. ${ }^{2}$ Nonlinear Dynamic Analysis Research Center, Department of Mathematics, Faculty of Applied Science, King Mongkut's University of Technology North Bangkok, Bangkok, 10800, Thailand. ${ }^{3}$ Department of Mathematics, University of loannina, loannina, 451 10, Greece.

\section{Acknowledgements}

The project was funded by the Deanship of Scientific Research (DSR), King Abdulaziz University, under grant no. 14-130-36-RG. The authors, therefore, acknowledge with thanks DSR's technical and financial support.

\section{Received: 13 September 2015 Accepted: 4 January 2016 Published online: 16 January 2016}

\section{References}

1. Kac, V, Cheung, P: Quantum Calculus. Springer, New York (2002)

2. Bangerezako, G: Variational q-calculus. J. Math. Anal. Appl. 289, 650-665 (2004)

3. Gasper, G, Rahman, M: Some systems of multivariable orthogonal q-Racah polynomials. Ramanujan J. 13, 389-405 (2007)

4. Ismail, MEH, Simeonov, P: q-Difference operators for orthogonal polynomials. J. Comput. Appl. Math. 233, 749-761 (2009)

5. Ahmad, B, Ntouyas, SK: Boundary value problems for q-difference inclusions. Abstr. Appl. Anal. 2011, Article ID 292860 (2011)

6. Annaby, MH, Mansour, ZS: q-Fractional Calculus and Equations. Lecture Notes in Mathematics, vol. 2056. Springer, Berlin (2012)

7. Graef, JR, Kong, L: Positive solutions for a class of higher order boundary value problems with fractional $q$-derivatives. Appl. Math. Comput. 218, 9682-9689 (2012)

8. Ahmad, B, Ntouyas, SK, Purnaras, IK: Existence results for nonlocal boundary value problems of nonlinear fractional q-difference equations. Adv. Differ. Equ. 2012, 140 (2012)

9. Zhou, W, Liu, H: Existence solutions for boundary value problem of nonlinear fractional q-difference equations. Adv Differ. Equ. 2013, 113 (2013)

10. Ferreira, RAC: Existence and uniqueness of solution to some discrete fractional boundary value problems of order less than one. J. Differ. Equ. Appl. 19, 712-718 (2013)

11. Ahmad, B, Nieto, JJ, Alsaedi, A, Al-Hutami, H: Existence of solutions for nonlinear fractional $q$-difference integral equations with two fractional orders and nonlocal four-point boundary conditions. J. Franklin Inst. 351, 2890-2909 (2014)

12. Agarwal, RP, Ahmad, B, Alsaedi, A, Al-Hutami, H: Existence theory for q-antiperiodic boundary value problems of sequential $q$-fractional integrodifferential equations. Abstr. Appl. Anal. 2014, Article ID 207547 (2014)

13. Ahmad, B, Ntouyas, SK: Fractional q-difference hybrid equations and inclusions with Dirichlet boundary conditions. Adv. Differ. Equ. 2014, 199 (2014)

14. Ferreira, RAC, Goodrich, CS: On positive solutions to fractional difference inclusions. Analysis 35, 73-83 (2015)

15. Zhao, J: Positive solutions for a class of $q$-fractional boundary value problems with $p$-Laplacian. J. Nonlinear Sci. Appl. 8, 442-450 (2015)

16. Tariboon, J, Ntouyas, SK: Quantum calculus on finite intervals and applications to impulsive difference equations. Adv. Differ. Equ. 2013, 282 (2013)

17. Agarwal, RP, Wang, G, Ahmad, B, Zhang, L, Hobiny, A: Successive iteration and positive extremal solutions for nonlinear impulsive $q_{k}$-difference equations. Adv. Differ. Equ. 2015, 164 (2015)

18. Tariboon, J, Ntouyas, SK, Agarwal, P: New concepts of fractional quantum calculus and applications to impulsive fractional q-difference equations. Adv. Differ. Equ. 2015, 18 (2015)

19. Granas, A, Dugundji, J: Fixed Point Theory. Springer, New York (2003) 